\title{
Organizing pneumonia secondary to cetuximab in a patient with colorectal cancer
}

\author{
Abdulaziz AlGhulayqah",2, Bader AIShamsan",3, Shouki Bazarbashi², Shamayel Mohammed ${ }^{4}$, \\ Abdullah Mobeireek ${ }^{2}$ \\ ${ }^{1}$ Department of Medicine, Qassim University; ${ }^{2}$ Department of Medicine, Section of Pulmonary, King Faisal Specialist \\ Hospital and Research Center, Riyadh; ${ }^{3}$ Oncology Center, Section of Medical Oncology, King Faisal Specialist \\ Hospital and Research Center, Riyadh; ${ }^{4}$ Department of Pathology, King Faisal Specialist Hospital and Research \\ Center, Riyadh, Saudi Arabia
}

\begin{abstract}
Organizing pneumonia (OP) may be idiopathic or secondary to a variety of causes including drugs. OP and other forms of pulmonary toxicity secondary to cetuximab, however, have been described rarely. It is paramount to recognize and differentiate OP from other common conditions that cancer patients are prone to such as infection and pulmonary embolism. A 69-year-old man with colorectal cancer received ten cycles of palliative chemotherapy [FOLFIRI (5-Fluorouracil, Leucovorin, Irinotecan) and cetuximab] with clinical and radiological response. He developed dyspnea following cycle 4 , then 6 weeks later presented with cough, fever, tachypnea, hypoxia, bilateral crackles and diffuse pulmonary shadows. He was started on antibiotics but his condition
\end{abstract}

Correspondence: Abdullah Mobeireek, P.O. Box 3354, MBC-46, Riyadh 11211, Saudi Arabia.

Tel. +966.114427493 - Fax: +966.114427499

E-mail: mobeireek@yahoo.com

Key words: Organizing pneumonia; cetuximab; colorectal cancer.

Acknowledgments: Authors are grateful to Dr. Abdullah Aljebreen and Dr. Fouad Al-Dayel for assistance in radiological and pathological material, respectively.

Conflict of interest: the authors declare no conflict of interest.

Contributions: AM, study concept and design, mentoring, manuscript revising and editing; SM, pathology review; AA, BA, SB, manuscript drafting revising and editing. All authors made a substantive intellectual contribution, and read and approved the final version of the manuscript.

Received for publication: 29 March 2019.

Accepted for publication: 4 August 2019.

${ }^{\circ}$ Copyright: the Author(s), 2019

Licensee PAGEPress, Italy

Monaldi Archives for Chest Disease 2019; 89:1074

doi: 10.4081/monaldi.2019.1074

This article is distributed under the terms of the Creative Commons Attribution Noncommercial License (by-nc 4.0) which permits any noncommercial use, distribution, and reproduction in any medium, provided the original author(s) and source are credited. deteriorated further. Cultures, including blood and bronchioalveolar lavage, grew no pathogens and molecular analysis and cytology for bacteria and viruses were negative. Trans-bronchial biopsy was consistent with organizing pneumonia. Treatment with corticosteroids resulted in dramatic clinical and radiological resolution with normalization of gas exchange and pulmonary function. Corticosteroids were stopped and he was restarted on FOLFIRI and remained well with no relapse over a year of follow up. Although pulmonary toxicity secondary to cetuximab is uncommon, it is important to recognize, as it may be associated with poor prognosis. To the best of our knowledge, this is the first report of OP attributed to cetuximab with histopathological evidence.

\section{Introduction}

Organizing pneumonia (OP) is a distinct clinical and pathological entity that is either idiopathic (formerly known as bronchiolitis obliterans with organizing pneumonia) or secondary to a variety of causes including autoimmune, pathogens, toxins and drugs $[1,2]$. Amongst the latter, newer biological agents used to treat cancer are increasingly reported to cause pulmonary toxicity [3]. The oncologist has today a wide range of options that can improve both the quality of life and survival in patients with this disease. Nonetheless, it is prudent to be vigilant of the potential adverse effects of such newer therapies. In this report, we describe a patient with colorectal cancer who developed OP after treatment with cetuximab.

\section{Case Report}

A 69-year-old male smoker was diagnosed with adenocarcinoma of the rectum located at $9-10 \mathrm{~cm}$ from the anal verge. The clinical stage was T3N2M1 (liver). He started palliative chemotherapy with FOLFOX (folinic acid, fluorouracil, and oxaliplatin) and bevacizumab. A follow-up computed tomography (CT) scan after cycle 4 revealed progression of the disease. Accordingly, his chemotherapy was changed to FOLFIRI (folinic acid, fluorouracil, and irinotecan) and cetuximab. Up to this point he reported no respiratory difficulties and his oxygen saturation and chest radiograph were within normal limits. CT scan evaluation after cycle 4 and 8 showed partial response. Following his $4^{\text {th }}$ chemotherapy cycle he started to have acne on his face and body, as well as mild dyspnea 
on exertion, which was investigated by chest radiography and echocardiogram were unremarkable and his dyspnea remained stable till cycle 10 following which he presented with worsening dyspnea associated with productive cough and fatigue. He denied other constitutional symptoms. On examination he was febrile reaching $38.1^{\circ} \mathrm{C}$ and he was in moderate respiratory distress with a respiratory rate of 30 per min. His oxygen saturation was $92 \%$ on 2 liters of supplemental oxygen. Chest examination showed bilateral inspiratory crackles. Laboratory test showed WBC of $4.73 \times 10^{9} / 1$, differential showed $68 \%$ neutrophils, $21.9 \%$ lymphocytes and no eosinophilia. His procalcitonin was $0.10 \mathrm{ng} / \mathrm{ml}$, hemoglobin $125 \mathrm{~g} / 1$ and other routine blood tests were unremarkable other than a low albumin level (27 g/l). He was admitted for evaluation and management. His chest X-ray showed diffuse bilateral airspace opacities (Figure 1A). Computed tomography of the chest showed bilateral patchy ground-glass opacities with smooth interlobular septal thickening, in keeping with the crazy paving pattern, there was small bilateral pleural effusion (Figure 1 B-D). Initially, bacterial pneumonia was suspected, and he was started empirically on piperacillin-tazobactam. However, his condition deteriorated further over the next two days following his admission.

All cultures including blood, sputum, and urine grew no pathogens. Tests to exclude autoimmune causes including ANA, rheumatoid factor, Anti-SSA (Ro) and anti-SSB (La) antibody were all negative. Flexible fiberoptic bronchoscopy performed, and bronchioalveolar lavage fluid revealed colorless slightly turbid liquid with WBC $7110^{\wedge} 6 / 1,37 \%$ of which were neutrophil and $31 \%$ lymphocyte. Quantitative respiratory culture, acid-fast bacilli (AFB), fungal culture, general viral screening, molecular analysis for atypical organism were all negative. Also, fluid cytology for atypical cells, stains for AFB, fungal and Pneumocystis jiroveci was negative. Trans-bronchial biopsy (TBB) from left upper lobe showed minimal interstitial inflammation with crushing artifact and mild interstitial fibrosis, organizing pneumonia, reactive pneumocytes and rare intra-alveolar macrophages (Figure 2).

Based on the biopsy results and negative cultures, he was commenced on parenteral steroids (methylprednisone $40 \mathrm{mg}$ every 6 h). Within the next $48 \mathrm{~h}$, he showed a dramatic improvement in his symptoms as well as his oxygenation. A repeat chest X-ray showed significant resolution of the bilateral airspace opacities (Figure $3 \mathrm{~A}$ ). CT chest was repeated one month following treatment which shows interval improvement of the previously noted bilateral diffuse ground-glass densities with some residual patchy densities and complete resolution of previously noted small bilateral pleural
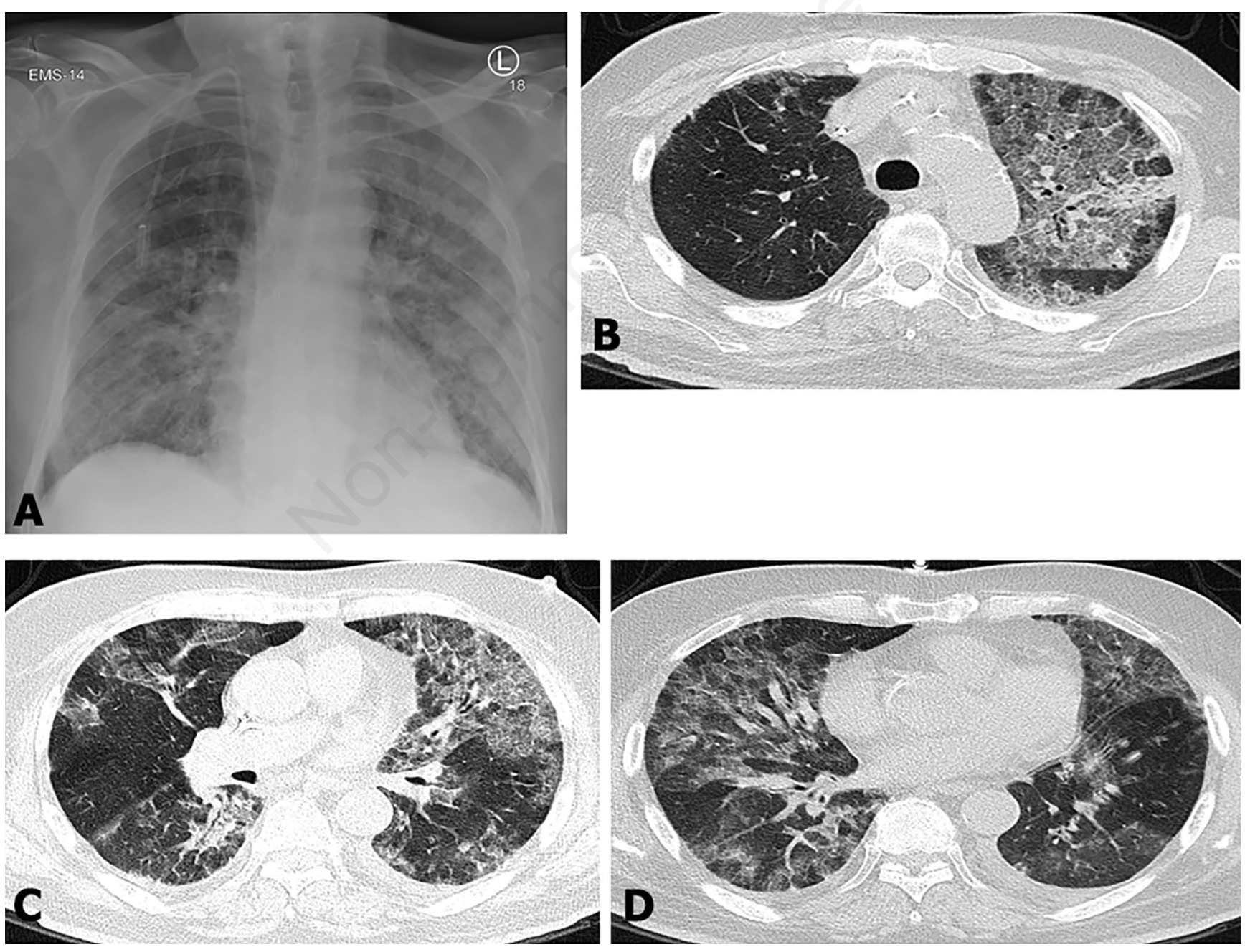

Figure 1. A) Chest X-ray showed diffuse bilateral airspace opacities. B-D) Selected coronal sections of chest CT showed bilateral patchy ground glass opacities with smooth interlobular septal thickening, in keeping with crazy paving pattern, there is small bilateral pleural effusion. 
effusion (Figure 3 B-D). His pulmonary function tests could not be performed initially because of respiratory distress but were within normal predicted values upon discharge.
He then received a tapering dose of oral prednisone which was eventually discontinued. Subsequently, he was re-challenged and received 12 cycles of FOLFIRI for his metastatic rectal cancer.
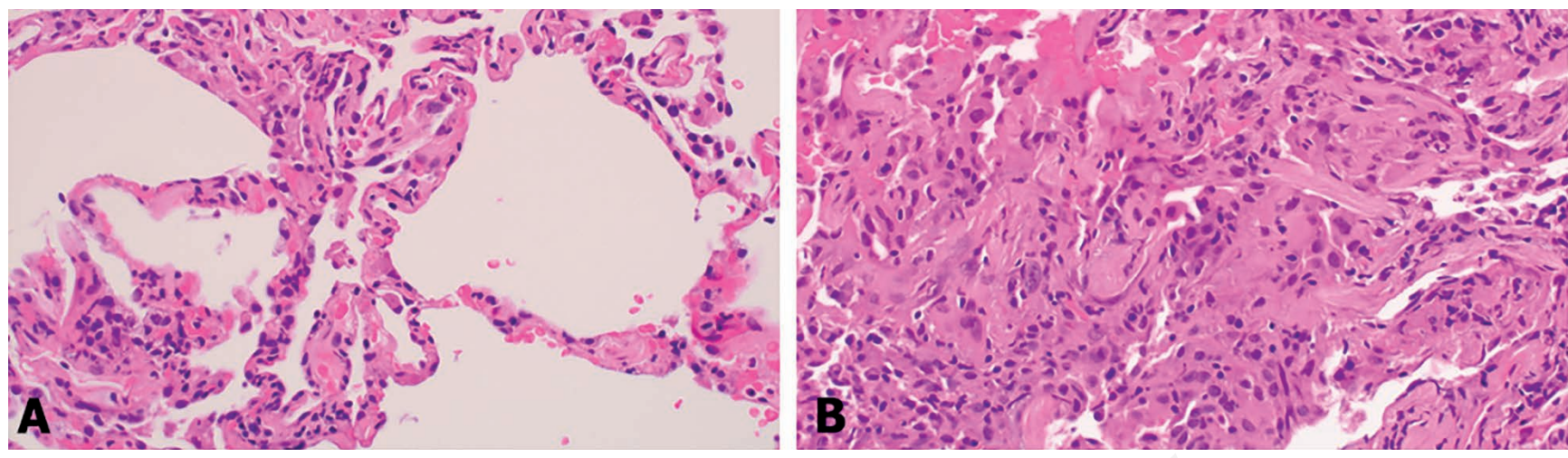

Figure 2. Hematoxylin and Eosin stain of the trans-bronchial biopsy from left upper lobe. A) minimal interstitial inflammation; magnification 20x. B) Crushing artifact, mild interstitial fibrosis, organizing pneumonia, reactive pneumocytes and rare intra-alveolar macrophages; magnification 40x.
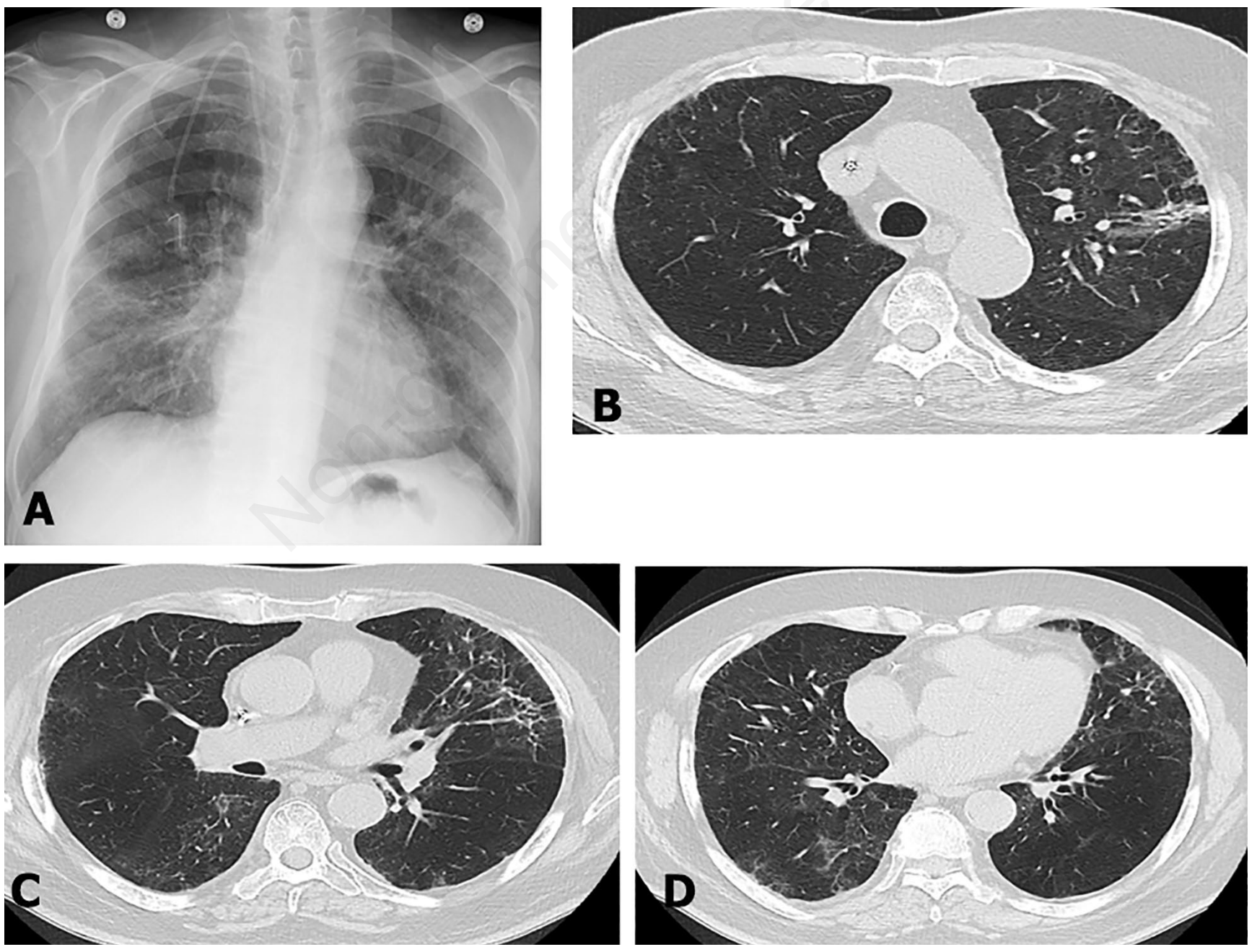

Figure 3. A) Chest X-ray showed significant improvement in bilateral airspace opacities. B-D) Selected coronal sections of chest CT showed interval improvement of the previously noted bilateral diffuse ground glass densities with some residual patchy densities with complete resolution of previously noted small bilateral pleural effusion. 
Restaging was done by MRI which revealed near-complete resolution of the lower/mid rectal tumor, no regional adenopathy, and there was also a complete resolution of the metastatic liver lesions with residual $1 \mathrm{~cm}$ lesion in segment 6 with no enhancement. He remains under close follow up for 12 months without relapse in his respiratory condition.

\section{Discussion}

Cetuximab is a recombinant chimeric IgG1 monoclonal antibody directed against the epidermal growth factor receptor (EGFR). EGFR is overexpressed in a broad range of human solid tumors, including colorectal cancer, head and neck cancer, NSCLC, pancreatic cancer, and breast cancer. Cetuximab binds to the extracellular domain of EGFR with nearly 10-fold higher affinity than normal ligands EGF and TGF- $\alpha$, which then results in inhibition of EGFR. This prevents both homo and heterodimerization of the EGFR, which leads to inhibition of autophosphorylation and inhibition of EGFR signaling resulting in inhibition of critical mitogenic and anti-apoptotic signals involved in proliferation, growth, invasion, metastasis and angiogenesis [4,5]. The mechanism of pulmonary toxicity is unclear. EGFR is expressed on type II pneumocytes and involved in alveolar wall repair. The inhibition of EGFR signaling might interrupt alveolar repair mechanisms. This may potentiate lung injury due to other causes, like preexisting interstitial lung disease (ILD), radiation therapy, prior lung injury, or other medications $[4,5]$. The most common side effects due to cetuximab are acneiform rash, infusion reactions, hypomagnesemia and gastrointestinal symptoms [3]. Pulmonary complications are rarely described in the literature, with only case reports describing adverse pulmonary reactions [3,6,7]. In one review, $10.3 \%$ of patients receiving cetuximab developed adverse pulmonary reactions; most common being dyspnea without any significant radiological changes [7]. Pneumonitis is reported but is considered a very rare pulmonary complication $[6,7]$.

Among reported cases, the age of the patients ranged between 55 and 78 years. The symptoms of organizing pneumonia were reported to develop between $4^{\text {th }}$ to the $11^{\text {th }}$ cycles $[3,8]$. Our patient reported his symptoms after $4^{\text {th }}$ cycle of FOLFIRI/Cetuximab regimen. FOLFIRI regimen on its own was reported to cause organizing pneumonia [9], but our patient was re-challenged with FOLFIRI regimen without relapse of the symptoms. Cough and shortness of breath with and without fever are the most common presentation among reported cases. The previous history of smoking and preexisting ILD were reported as risk factors $[3,6]$. In addition, previous chemotherapy might enhance progression of cetuximab-related pulmonary toxicity. He had low serum albumin level which was noted to be another predisposing factor for developing pulmonary complication secondary to biological treatment [3].

The initial diagnostic workup should exclude infectious etiologies. Bronchoalveolar lavage in two out of three previously reported cases, as well as our patient, was performed and all the microbiologic and cytologic studies were negative. Additionally, there was no eosinophilia to suggest eosinophilic pneumonia. Furthermore, our patient had a transbronchial biopsy which showed organizing pneumonia. A surgical lung biopsy is the gold standard for diagnosis of OP, but not without the risk of complications especially for hypoxemic patients. A TBB with the compatible clinical picture (including imaging and response to steroids) is considered to be an adequate tool; Poletti et al. performed TBB in 32 patients with suspected organizing pneumonia; the sensitivity, specificity, positive predictive value and negative predictive values of TBB were $64 \%, 86 \%, 94 \%$, and $40 \%$, respectively [10].

Corticosteroids are the mainstay for the treatment of OP. All reported cases, as well as our patient, showed clinical improvement after receiving corticosteroids. However, long-term prognosis is related to the primary disease; two of the reported cases died from complication of the primary malignancy, and the third was reported to be still surviving $[3,8]$. Our patient is still alive after a follow-up period of 12 months without relapse of his respiratory symptoms.

In conclusion, pulmonary toxicity secondary to cetuximab is uncommon but important to recognize and manage as it may be associated with poor outcome. This case report documents clinically, radiologically and, for the first time, histopathologically proven OP that is related to cetuximab. Physicians need to be aware of drug-related pulmonary toxicity with the increasing use of newer agents in cancer therapy.

\section{References}

1. Al-Saghir A, Mobeireek A. Bronchiolitis obliterans organizing pneumonia. Saudi Med J 2004;25:557-65.

2. Alsaghir AH, Al-Mobeireek AF, Al-Jahdali H, et al. Bronchiolitis obliterans organizing pneumonia: experience at three hospitals in Riyadh. Ann Saudi Med 2007;27:32-5.

3. Achermann Y, Frauenfelder T, Obrist S, et al. A rare but severe pulmonary side effect of cetuximab in two patients. BMJ Case Rep 2012;2012. pii: bcr032012597.

4. Miettinen PJ, Warburton D, Bu D, et al. Impaired lung branching morphogenesis in the absence of functional EGF receptor. Dev Biol 1997;186:224-36.

5. Hardie WD, Prows DR, Leikauf GD, Korfhagen TR. Attenuation of acute lung injury in transgenic mice expressing human transforming growth factor- $\alpha$. Am J Physiol 1999;277: L1045-50.

6. Satoh T, Gemma A, Kudoh S, et al. Incidence and clinical features of drug-induced lung injury in patients with advanced colorectal cancer receiving cetuximab: results of a prospective multicenter registry. Jpn J Clin Oncol 2014;44:1032-9.

7. Hoag JB, Azizi A, Doherty TJ, et al. Association of cetuximab with adverse pulmonary events in cancer patients: a comprehensive review. J Exp Clin Cancer Res 2009;28:113.

8. Chua W, Peters M, Loneragan R, Clarke S. Cetuximab-associated pulmonary toxicity. Clin Colorectal Cancer 2009;8:118-20.

9. Lee YJ, Kim JH, Kim SW, et al. A case of organizing pneumonia associated with FOLFIRI chemotherapy. Tuberc Respir Dis (Seoul) 2014;77:262-5.

10. Poletti V, Cazzato S, Minicuci N, et al. The diagnostic value of bronchoalveolar lavage and transbronchial biopsy in cryptogenic organizing pneumonia. Eur Respir J 1996;9:2513-6. 